\title{
Facial Muscle Weakness, CTCAE
}

National Cancer Institute

\section{Source}

National Cancer Institute. Facial Muscle Weakness, CT CAE. NCI Thesaurus. Code C143472.

A disorder characterized by a reduction in the strength of the facial muscles. 